\title{
Las acciones de marketing interno y el compromiso organizacional en hoteles de cuatro y cinco estrellas
}

Ações de marketing interno e comprometimento organizacional em hotéis de quatro e cinco estrelas

Internal marketing actions and organizational commitment in four and five star hotels

(9) Laura Monserrat Luna Valderrabano

- Maestra en Mercadotecnia

- $\quad$ Docente por asignatura en la Facultad de Ciencias de la Salud de la Universidad Autónoma de Baja California

- llunavalderrabano@gmail.com

(9) Nina Alejandra Martínez Arellano

- Doctora en Ciencias y Humanidades para el Desarrollo Interdisciplinario

- Coordinadora de la Maestría en estudios socioculturales. En la Facultad de Ciencias Administrativas y Sociales de la Universidad Autónoma de Baja California.

- nina.martinez@uabc.edu.mx 


\section{ANO 16 • NÚMERO 30 • 1ํㅗ․ SE. 2019 • ORGANICOM \\ LAS ACCIONES DE MARKETING INTERNO Y EL COMPROMISO ORGANIZACIONAL EN HOTELES DE CUATRO Y CINCO ESTRELLAS}

\section{Resumen}

Esta investigación parte de la teoría del marketing interno y sus efectos en el desarrollo organizacional, con una perspectiva en el desempeño de los miembros de la organización (clientes internos), identificando las acciones que contribuyen a fortalecer su compromiso organizacional. Así como analizando que acciones son las que influyen directamente en la calidad de la prestación de los servicios que presta cada empresa hotelera hacia sus clientes externos. Presenta un análisis inicial de la relación que existe entre las acciones de marketing interno y el compromiso organizacional de los clientes internos que laboran en hoteles de cuatro y cinco estrellas. Para alcanzar dicho objetivo, se creó un instrumento cuantitativo denominado cuestionario Internal marketingcommitment, compuesto por 40 ítems que evalúan el nivel de compromiso organizacional que tienen los clientes internos, así como su percepción acerca de las acciones de marketing interno en la empresa; a través de las cuales se busca medir cómo las acciones de marketing interno contribuyen a fortalecer el compromiso organizacional en los hoteles. Los resultados de la aplicación del instrumento muestran que existe una correlación positiva entre ambas variables, lo que indica que a una mayor percepción de acciones de marketing interno, mayor es el nivel de compromiso organizacional que presentan los clientes internos.

\section{PALABRAS CLAVE: MARKETINGINTERNO・COMPROMISO ORGANIZACIONAL・SECTOR HOTELERO.}

\section{Resumo}

Esta pesquisa se baseia na teoria do marketing interno e seus efeitos no desenvolvimento organizacional. Com uma perspectiva sobre 0 desempenho dos membros da organização (clientes internos), identifica as ações que contribuem para fortalecer seu comprometimento organizacional, além de analisar quais ações influenciam diretamente na qualidade da prestação dos serviços que cada empresa hoteleira oferece aos seus clientes externos. Apresenta uma análise inicial da relação entre as ações de marketing interno e o comprometimento organizacional dos clientes internos que atuam em hotéis de quatro e cinco estrelas. Para atingir esse objetivo, foi criado um instrumento quantitativo chamado questionário Internal marketing-commitment, composto por 40 itens que avaliam o nível de comprometimento organizacional dos clientes internos, bem como sua percepção das ações internas de marketing da empresa, por meio do qual se busca medir como as ações de marketing interno contribuem para o fortalecimento do comprometimento organizacional nos hotéis. Os resultados da aplicação do instrumento mostram que existe uma correlação positiva entre as duas variáveis, o que indica que quanto maior a percepção das ações de marketing interno, maior o nível de comprometimento organizacional que os clientes internos apresentam.

\section{PALAVRAS-CHAVE: MARKETING INTERNO・COMPROMISSO ORGANIZACIONAL・SETOR HOTELEIRO}

\section{Abstract}

This research is support on the theory of internal marketing and its effects on organizational development. With a perspective on the performance of the members of the organization (internal clients), identifying the actions that contribute to strengthen their organizational commitment. As well as analyzing wich actions are those that directly influence the quality of the provision of the services that each hotel company provides to its external clients. Presenting an inicial analysis of the relationship between internal marketing actions and the organizacional commitment of internal customers working in four and five stars hotels. To achieve this objective, a quantitative instrument called Internal questionnaire marketing-commitment was designed. It consist of 40 items that assess the level of organizational commitment with internal customers have, as well as their perception on the company's internal marketing actions, through which it sought to measure how the actions of internal marketing contribute to strengthening organizational commitment in hotels. The results of the application of the instrument show that there is a positive correlation between the two variables, indicating that the greater the perception of internal marketing activities, the greater the level of organizational commitment of internal customers. 


\section{ANO 16 • NÚMERO 30 • 1ํSEM. 2019 • ORGANICOM \\ LAS ACCIONES DE MARKETING INTERNO Y EL COMPROMISO ORGANIZACIONAL EN HOTELES DE CUATRO Y CINCO ESTRELLAS}

\section{INTRODUCCIÓN}

El concepto de marketing interno ha evolucionado gracias a los estudios que han realizado muchos investigadores (Berry; Hensel; Burke, 1976; Grönroos, 1994; Kobli; Jaworski, 1990), quienes lo han definido como un esfuerzo planteado para realizar una aproximación de marketing que ayude a crear un cambio para alinear y coordinar de manera interfuncional los esfuerzos de la organización y satisfacer las necesidades de sus clientes internos. Según la literatura revisada y de acuerdo al enfoque desde el cual se aborda, al cliente interno se le denomina de diferentes maneras - trabajador, empleado, capital humano o talento humano -. Pero para esta investigación se utilizará el término "cliente interno", a partir de la perspectiva de la mercadotecnia. Mendoza Moheno, Hernández Calzada y Tabernero Urbieta (2011) señalan en sus estudios que el enfoque de marketing interno reconoce el potencial del empleado para desarrollar una ventaja para su organización, con la implementación de estrategias corporativas, con la intención de satisfacer las necesidades motivando la satisfacción y el compromiso organizacional de los clientes internos.

A partir de estas investigaciones, se desarrolló la orientación del marketing interno (OMI) a mediados de la década de los 90 , cuando estudiosos del tema (Bohnenberger, 2005; Grönroos, 1994; Kohli; Jaworski, 1990; Rafiq; Ahmed, 2000) expresaron que esta orientación tiene su origen a raíz de la conceptualización del marketing de servicios, en que las acciones estaban enfocadas hacia la satisfacción de las necesidades del cliente externo de la empresa. Para algunos autores (Berry; Hensel; Burke, 1976; Coca Carasila, 2008), el marketing interno es una variación de la mercadotecnia en que se considera a los empleados como clientes internos, donde se ven los puestos de trabajo como productos internos que satisfacen las necesidades y deseos de los mismos, mientras que consecutivamente se consiguen los objetivos de la organización.

En otras palabras, el marketing interno consiste en atraer, desarrollar, motivar y retener a los clientes internos que forman parte de una organización; estos deben estar calificados para cubrir las posiciones laborales disponibles - productos que satisfagan sus necesidades - (Parasuraman, 1997). Esto con la finalidad de crear una ventaja que distinga a su servicio; una estrategia clave para conseguir los mejores resultados en el sector hotelero, pues para generar mayor valor al cliente externo hay que tener clientes internos satisfechos y comprometidos (Ruizalba Robledo; Vallespín Arán; Pérez-Aranda, 2015).

El marketing interno requiere de un trabajo integral que equilibre los enfoques interno y externo de la organización, desde las perspectivas de la relación cliente interno - organización -, pues a partir de esto se busca el beneficio mutuo. Este proceso necesita establecer una relación entre el cliente interno y la organización, que se da a partir del compromiso organizacional que surge entre ambas partes (Mendoza Moheno; Hernández Calzada; Tabernero Urbieta, 2011). Por consecuencia, estudios recientes (Bohnenberger, 2005; Silva; Lira, 2014) señalan que las acciones de marketing interno promueven la motivación y el compromiso de los clientes internos con los objetivos organizacionales, por lo que se considera que esta orientación de marketing trabaja en conjunto con los mandos medios de la administración de las empresas, pues es por medio de estos que se puede llevar a cabo dichos procesos organizacionales de forma dinámica y eficiente.

Si bien el marketing interno puede verse relacionado con variables como gestión del conocimiento, aprendizaje organizacional, desarrollo de innovación, calidad en el servicio, talento humano, entre otras; ha resultado de mucho interés para esta investigación el analizar la relación que existe entre las acciones de marketing interno y el compromiso organizacional de los clientes internos en la organización.

El marketing internose define entonces como una filosofía (Antunes y Antunes, 2012) basada en tratar al talento humano como cliente interno, con énfasis en la creación de estrategias que impulsen en los clientes internos su compromiso motivándolos a que se desempeñen alineados para alcanzar los objetivos organizacionales. A través de distintas estrategias de comunicación 
interna que motiven el compromiso, fidelidad y orgullo del cliente interno con la organización (Brandão, 2018; Santos, 2018), para lo cual es necesario que el equipo de trabajo conozca y asimile como propios los objetivos de la organización.

Por lo anterior, es necesario definir al compromiso organizacional como el grado en el que un cliente interno se identifica con la organización y desea seguir participando activamente en ella. Es decir, un conjunto de vínculos que mantienen a un sujeto apegado a una organización en particular (Allen; Meyer, 1996; Arciniega, 2002; Pérez Vera; De la Garza Carranza, 2008). Esta relación surge entre el individuo (cliente interno) y su organización (su filosofía empresarial como los objetivos, políticas y valores), y consiste en la intensidad de la participación y la identificación que tenga el cliente interno con su organización (Hellriegel; Slocum Junior, 2004; Mowday; Steers; Porter, 1979).

El compromiso organizacional, entonces, es comprendido como el sentido de pertenencia o lealtad que la organización despierta en sus clientes internos (Geyskens; Steenkamp; Scheer; Kumar, 1996; Ruizalba Robledo; Vallespín Arán; Pérez-Aranda,2015). Este compromiso constituye un constructo multidimensional, gestor de cambios, que impulsa la transformación empresarial y fomenta un ambiente organizacional de transparencia y confianza (Meyer; Herscovitch, 2001; Zapata Palacios; Gómez Quijano, 2016). Allen y Meyer (1996) en sus investigaciones señalan que el compromiso puede ser identificado como afectivo, normativo o de continuidad, de acuerdo a las motivaciones de cada cliente interno (Peterson; Puia; Suess, 2003).

Hasta el momento no se ha establecido con claridad cuál es la relación que tienen las acciones de marketing interno y el compromiso organizacional de los clientes internos en hoteles de cuatro y cinco estrellas, por lo que llevar a cabo una investigación de este tipo beneficiaría la caracterización de las acciones de marketing interno que mejoran el compromiso organizacional en dichas empresas, lo que favorecería tanto para las organizaciones que brindan este servicio en la localidad como al cliente interno que labora en éstas. En este sentido, el objetivo de la presente investigación se concentra en evaluar la relación que tienen las acciones de marketing interno, en hoteles, con el compromiso organizacional de los clientes internos de estas empresas, desde la hipótesis de que estas acciones contribuyen a fortalecer el compromiso organizacional en hoteles de cuatro y cinco estrellas.

\section{MÉTODO}

Para explorar la correlación entre estas variables, se diseñó una metodología cuantitativa que consideró el diseño de un instrumento denominado Internal marketing-commitment, que se compone de 40 ítems, validado por medio de pruebas estadísticas como la correlación de Pearson y el alpha de Cronbach. A continuación, se detalla la estructura del instrumento, así como los pormenores de su aplicación.

\section{Materiales e instrumentos}

En la presente investigación, se creó un instrumento cuantitativo en forma de encuesta (Cuestionario Internal marketingcommitment), con base en una escala de Likert de 5 opciones, donde 5 es totalmente de acuerdo y 1 totalmente en desacuerdo. Su objetivo es evaluar las acciones de marketing interno y el compromiso organizacional, según la percepción de los clientes internos, en las organizaciones hoteleras de cuatro y cinco estrellas. Para tal propósito, se dividió en las siguientes dimensiones (ver Cuadro 1): 
ANO 16 • NÚMERO 30 • 1은. 2019 • ORGANICOM

LAS ACCIONES DE MARKETING INTERNO Y EL COMPROMISO ORGANIZACIONAL

EN HOTELES DE CUATRO Y CINCO ESTRELLAS

Cuadro 1: Dimensiones del cuestionario Internal marketing-commitment

\begin{tabular}{|c|c|c|c|}
\hline Variable & Dimensiones & Indicadores & Subindicadores \\
\hline \multirow{8}{*}{ Marketing interno } & \multirow{4}{*}{ Adecuación al trabajo } & \multirow{4}{*}{$\begin{array}{l}\text { Formación } \\
\text { (8 ítems) }\end{array}$} & Inducción \\
\hline & & & Capacitación \\
\hline & & & Sucesión \\
\hline & & & Evaluación del desempeño \\
\hline & \multirow{4}{*}{ Motivación } & \multirow{4}{*}{$\begin{array}{l}\text { Reconocimiento } \\
\text { (8 ítems) }\end{array}$} & Eventos de reconocimiento \\
\hline & & & Becas \\
\hline & & & Promociones \\
\hline & & & Becas - incentivos \\
\hline \multirow{7}{*}{ (variable independiente) } & \multirow{7}{*}{ Comunicación interna } & \multirow{2}{*}{$\begin{array}{l}\text { Canales } \\
\text { (4 ítems) }\end{array}$} & Formales \\
\hline & & & Informales \\
\hline & & \multirow{3}{*}{$\begin{array}{l}\text { Flujos de comunicación } \\
\text { (6 ítems) }\end{array}$} & Vertical ascendente \\
\hline & & & Vertical descendente \\
\hline & & & Horizontal \\
\hline & & \multirow{2}{*}{$\begin{array}{l}\text { Información } \\
\text { (4 ítems) }\end{array}$} & Reuniones de trabajo \\
\hline & & & Tratamiento de quejas \\
\hline \multirow{5}{*}{$\begin{array}{l}\text { Compromiso organizacional } \\
\text { (variable dependiente) }\end{array}$} & \multirow{2}{*}{ Nivel de identificación } & \multirow{2}{*}{$\begin{array}{c}\text { Grado de identificación } \\
\text { (4 ítems) }\end{array}$} & Conocimiento \\
\hline & & & Integración \\
\hline & \multirow{3}{*}{ Satisfacción laboral } & \multirow{3}{*}{$\begin{array}{c}\text { Grado de satisfacción } \\
\text { laboral } \\
\text { (6 ítems) }\end{array}$} & Seguridad \\
\hline & & & Estabilidad \\
\hline & & & Trabajo colaborativo \\
\hline
\end{tabular}

Fuente: Elaborado por las autoras.

El instrumento cuenta con 40 ítems, divididos por dimensiones, los que permiten medir tanto la variable independiente (marketing interno) como la dependiente (compromiso organizacional).

\section{Validez}

La validez de contenido del instrumento se determinó mediante la validez por expertos, el cuestionario Internal marketingcommitment se envió para la revisión con investigadores, entre los que se encuentran: la Dra. Edith Molina Carmona (PTC investigadora, Secretaria académica de la BUAP y responsable del cuerpo académico Comunicación y sociedad), la Dra. Hilda Gabriela Hernández Flores (PTC Investigadora de la BUAP), la Dra. Paola Eunice Rivera (PTC Investigadora de la BUAP), la Dra. María Guadalupe Curro Lau (PTC Investigadora de la BUAP) y por el Dr. Isaac Uribe Alvarado (PTC Investigador de la UCOL).

Una vez que los jueces lo revisaron, se determinó la validez mediante el análisis de correlación de Pearson $(r=0,595)$. En tanto que la validez convergente se determinó al aplicar de manera conjunta el inventario de Bohnenberger (2005) - que mide marketing interno y compromiso organizacional -, y se obtuvo valores similares al del cuestionario Internal marketingcommitment. En cuanto a la confiabilidad, se determinó el índice de consistencia interna del instrumento mediante del alpha de Cronbach cuyo resultado fue de 0,96 para el total de los ítems. 


\section{Participantes}

Los resultados de las pruebas descriptivas aplicadas a la base de datos, obtenida en el trabajo de campo realizado en dos hoteles de cuatro y cinco estrellas, en Ensenada Baja California, (denominados para este estudio como SN y EC) donde se aplicó a 157 clientes internos que laboran en dichas empresas (84 de la SN y 73 de EC) la encuesta denominada "internal marketingcommitment". El grupo de participantes estuvo integrado por un total de 157 personas ( 65 mujeres y 92 hombres) (ver tabla 1).

Tabla 1: Género de los participantes

\begin{tabular}{|c|c|c|}
\hline & $\mathrm{N}$ & $\%$ \\
\hline \multicolumn{3}{|l|}{ Género } \\
\hline Femenino & 65 & 41.4 \\
\hline Masculino & 92 & 58.6 \\
\hline
\end{tabular}

Fuente: Elaborado por las autoras.

La media de edad del grupo es de 31 años, con una desviación estándar de 11.216. La edad de los participantes fluctúa entre los 17 y 66 años. Para la selección de la muestra de la prueba piloto, se tuvieron en cuenta los siguientes criterios:

\section{- Criterios de inclusión}

- Estar trabajando en el área de atención a clientes en un hotel de cuatro o cinco estrellas.

- Tener más de 6 meses laborando en dicha empresa.

\section{- Criterios de exclusión}

- Que su trabajo dentro de la organización no tenga contacto directo con clientes externos.

- Que no forme parte del personal de hotel (outsourcing).

\section{Muestreo}

Para la aplicación del instrumento se consideró pertinente el tomar una muestra no probabilística por conveniencia, donde la unidad de análisis se conformó por los clientes internos de dos hoteles de cuatro y cinco estrellas (SN y EC), que forman parte del universo a estudiar (cuatro hoteles). La selección de la muestra dependió de la disponibilidad y aceptación para participar, pues por las condiciones del universo se requiere de una cuidadosa y controlada elección de casos con ciertas características especificadas en el planteamiento (Hernández Sampieri; Fernández Collado; Baptista Lucio, 2010; Molina Carmona; Hernández, 2006).

\section{Procedimiento}

A continuación, se muestran los pasos que se siguieren durante la aplicación del instrumento Internal marketing-commitment.

1. Construcción del instrumento cuantitativo. 
2. Aplicación de prueba piloto a 29 personas. Para la aplicación de la prueba piloto, se llevó el instrumento impreso, plumas y tablas para que los participantes pudieran responder los cuestionarios de manera cómoda.

3. Análisis de resultados de la prueba piloto.

4. Aplicación de cuestionario a 157 participantes Se solicitó permiso en las organizaciones para aplicar a los clientes internos que laboran en las áreas de servicio de atención al cliente.

5. Se aplicó el instrumento cuantitativo.

6. El tiempo de aplicación fue de aproximadamente 20 minutos por participante, por lo que se tuvo que formar grupos de cerca de 7 personas, para explicar el instrumento y esperar sus respuestas.

7. Creación de la base de datos en el programa Statistical Package for the Social Sciences.

8. Análisis e interpretación de la base de datos con pruebas estadísticas en SPSS.

9. Reporte de resultados de la aplicación del instrumento cuantitativo.

\section{Pruebas estadísticas}

Para determinar la validez de contenido, se aplicó análisis de correlación de Pearson, así mismo para comprobar la confiabilidad se utilizó un análisis Alpha de Cronbach. La rde Pearson ayudó a establecer la relación existente entre el compromiso y el marketing interno (Triola, 2009).

\section{RESULTADOS}

La muestra tuvo una edad promedio de 37 años, con una desviación estándar ( $\sigma$ ) de 13,18. De estos, el 44,8\% de los participantes de la prueba fueron hombres, mientras que el $55,2 \%$ fueron mujeres. De los cuales el $48,3 \%$ se identificaron su estado civil como solteros, el 34,5\% estaban casados o en una relación estable, en tanto que los demás estados civiles (viudo, divorciado) sumaron el 17,2\%. En el estudio se encontró que el 55,2\% de los encuestados tienen hijos.

Las respuestas de los participantes (trabajadores en el área de servicios y atención al cliente) señalaron que el 37,9\% de los encuestados tienen más de 6 años de experiencia en este campo de actuación, mientras que el 27,6\% manifestaron tener sólo un año de experiencia laboral en dichas áreas.

En el análisis de frecuencias, la media de marketing interno fue de 117,5, con un mínimo de 86 y un máximo de 149. Mientras que la media de compromiso organizacional fue de 39,5, con un mínimo de 26 y un máximo de 50. Lo que indica que hay un resultado positivo entre la percepción de las acciones de marketing interno que tienen los clientes internos y el nivel de compromiso organizacional que estos muestran (ver Tabla 2): 
Tabla 2: Percentiles de las variables

\begin{tabular}{l|c|c|c}
\hline \multicolumn{4}{c}{ Estadísticos } \\
\hline \multicolumn{2}{c}{} & Marketing interno & $\begin{array}{c}\text { Compromiso } \\
\text { organizacional }\end{array}$ \\
\hline \multirow{2}{*}{$\mathrm{N}$} & Válido & 29 & 29 \\
\cline { 2 - 4 } & Perdidos & 0 & 0 \\
\hline Media & & 117.5172 & 39.5862 \\
\hline Mínimo & & 86.00 & 26.00 \\
\hline Máximo & 25 & 149.00 & 50.00 \\
\hline \multirow{2}{*}{ Percentiles } & 50 & 101.5000 & 35.0000 \\
\cline { 2 - 4 } & 75 & 115.0000 & 39.0000 \\
\hline
\end{tabular}

Fuente: Elaborado por las autoras.

Los análisis de las pruebas aplicadas a los datos obtenidos de la encuesta revelan una similitud en la relación que tienen con respecto al nivel de compromiso organizacional con las acciones de marketing interno. Es decir, donde el nivel de marketing interno es alto, el compromiso organizacional también se presenta alto (ver Figura 1).

Figura 1: Niveles de marketing interno y compromiso organizacional

Marketing interno y compromiso organizacional

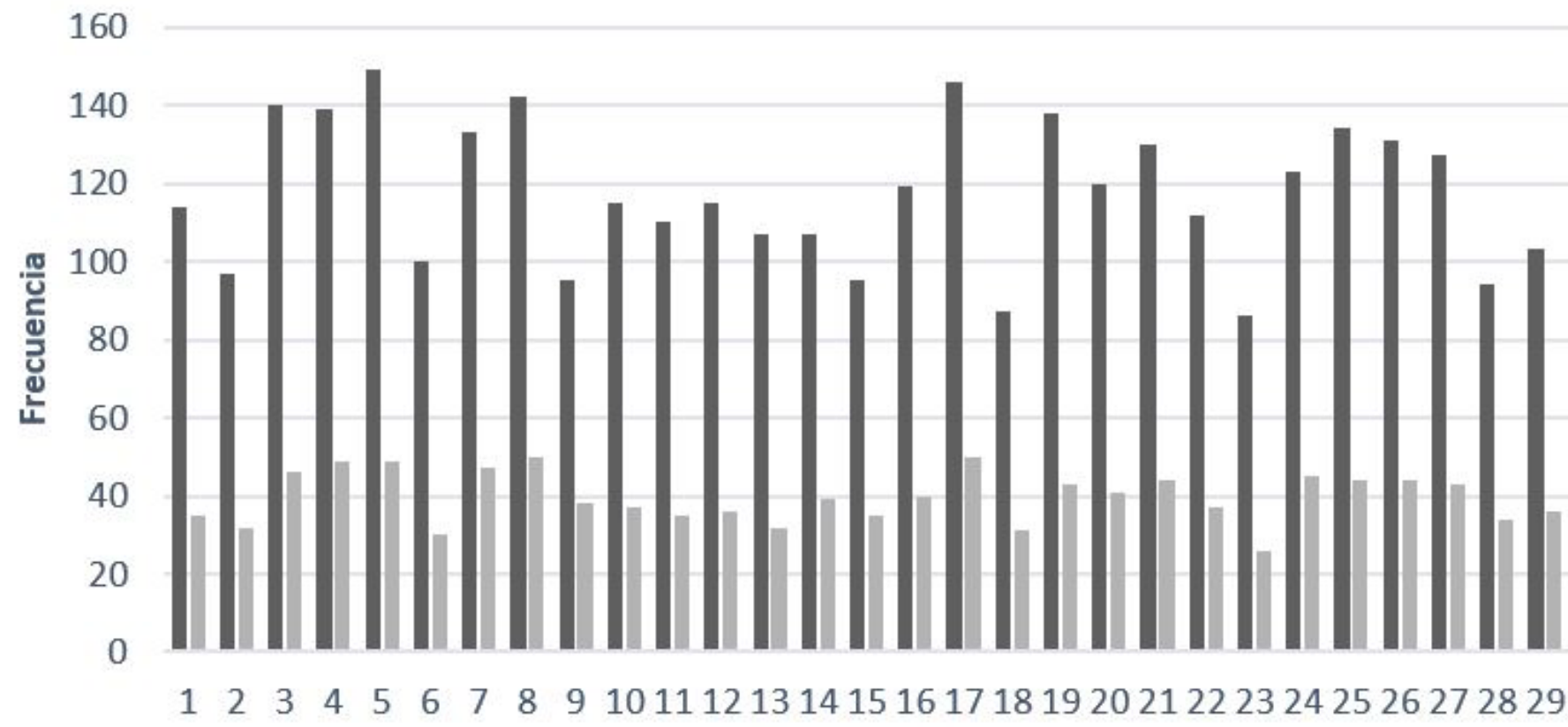

Encuestados

$\because \mathrm{MI}=\mathrm{CO}$

Fuente: Elaborado por las autoras.

En el análisis de correlación de Pearson, el resultado indicó que hubo una correlación positiva entre las variables $(r=0,92$, $p<0,01)$. Es decir, mayores son niveles de acciones de marketing interno en las organizaciones, mayores son los niveles de compromiso organizacional que se presentan, como se puede observar en el diagrama de dispersión que se muestra a continuación (ver Figura 2): 
Figura 2: Diagrama de correlación de Pearson

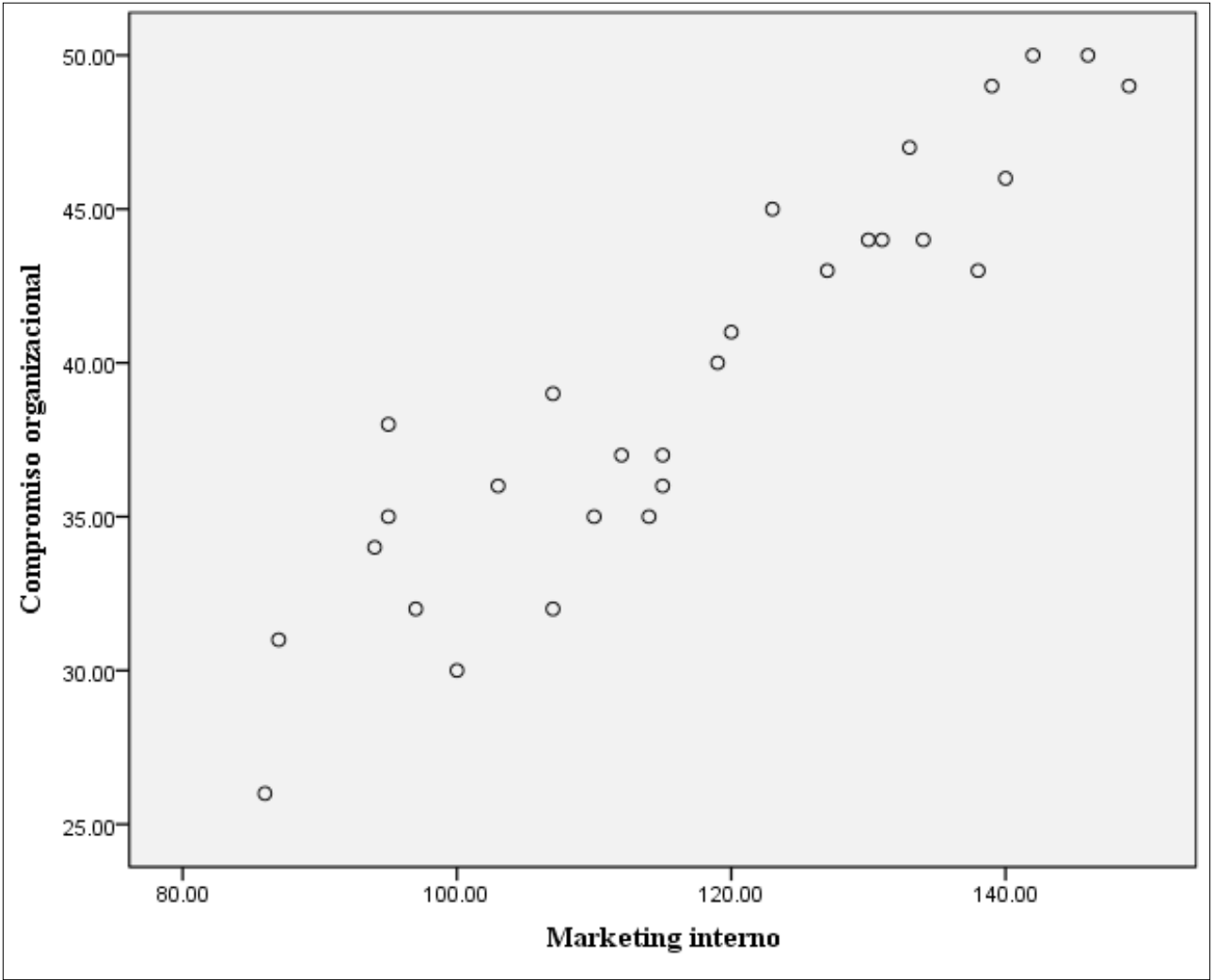

Fuente: Elaborado por las autoras mediante SPSS.

\section{DISCUSIÓN}

Los resultados obtenidos en la aplicación del instrumento Internal marketing-commitment son consistentes con lo reportado por Pérez Vera y De la Garza Carranza (2008), quienes en su estudio sobre comportamiento organizacional y satisfacción laboral destacan que en toda organización en la que sus empleados tienen un fuerte compromiso, la empresa tiende a beneficiarse de muchas maneras, entre otras cosas con: un decremento de los índices de rotación de personal, un aumento en la productividad, mayor cumplimiento de resultados, un notorio incremento en la motivación y en la satisfacción de los empleados.

Esto podría deberse a que el marketing interno afecta el compromiso organizacional de los clientes internos (Allen; Meyer, 1996; Berry; Hensel; Burke 1976; Grönroos, 1994). Y a su vez el compromiso organizacional influye sobre la selección de acciones de marketing interno que deberán utilizarse en cada organización, según sus necesidades específicas (Bekin, 2004; Silva; Lira, 2014).

Esta investigación se ha planteado por medio de un estudio no experimental que permitió definir la relación que existe entre las acciones de marketing interno y el compromiso organizacional; al igual que Lozano (2016) quien en su estudio aplica la metodología de diseño no experimental y el análisis correlacional aplicado al estudio de los factores que intervienen en el estudio del marketing interno y sus efectos en el aprendizaje organizacional. Así mismo Castillo-Vergara, Alvarez-Marin, Matsuda Oteiza, Alvarado y Codoceo (2016) desarrollaron una investigación acerca del marketing interno y sus efectos en el desarrollo de la innovación en la asociación gremial gastronómica de Peñuelas, en la región de Coquimbo en Chile, donde realizaron un estudio cuyos resultados señalaron la asociación que existe entre sus recursos y el marketing interno; consiguiendo una valoración de la implicación de sus clientes internos, de primera línea, y una valoración de la participación activa de sus clientes externos, todo esto por medio de un análisis de varianza de un factor, un análisis factorial exploratorio y un análisis de coeficiente de correlación Spearman. 


\section{ANO 16 • NÚMERO 30 • 1ํㅗ․ SE. 2019 • ORGANICOM \\ LAS ACCIONES DE MARKETING INTERNO Y EL COMPROMISO ORGANIZACIONAL EN HOTELES DE CUATRO Y CINCO ESTRELLAS}

En el presente estudio se realizó un muestreo no probabilístico por conveniencia, dadas las características de los participantes, al igual que Villarejo Ramos (2008), quien en su estudio realizó la propuesta de un modelo teórico, en que las hipótesis fueron validadas por medio de un método de muestreo no probabilístico entre una población formada por el 58,6\% de mujeres desde una muestra total de 309 individuos. En dicho análisis se revisaron los constructos de: Calidad de servicio, satisfacción, compromiso organizacional, confianza y comportamientos de lealtad.

Mientras que el presente estudio permitió la aplicación del cuestionario Internal marketing-commitment a 157 participantes, pertenecientes a dos de las organizaciones hoteleras de cuatro y cinco estrellas en la Ciudad de Ensenada, Baja California, México. Mismas que forman parte del universo de 6 empresas con estas clasificaciones en dicha zona.

Cabe señalar que esta aplicación permitirá profundizar en futuros análisis en el desarrollo de un modelo de ecuaciones estructurales, para establecer relaciones entre los constructos y beneficiar a la investigación con el poder predictivo del modelo estructural.

\section{CONCLUSIÓN}

Tras el análisis de los resultados preliminares por el análisis estadísticos de los datos obtenidos se infiere que las acciones de marketing interno percibidas por los clientes internos de la organización tienen una relación con los niveles de compromiso organizacional que muestran los miembros de la organización. Los resultados obtenidos en este piloto indican que es posible la comprobación de la hipótesis inicial, al demostrar la existencia de esta relación, de donde se parte al análisis de las condiciones en las que ésta se da, en relación a la manera en que las acciones de marketing interno tienen un efecto positivo en el compromiso organizacional.

La importancia de la relación entre el marketing interno y el compromiso organizacional radica en el grado de identificación y satisfacción que el cliente interno desarrolla por su organización, comprometido con la filosofía, los valores y los objetivos organizacionales, esto por medio de los contratos psicológicos (Lu; Capezio; Restubog; García; Wang, 2016) y la identificación organizacional de los empleados, que se basa en la correlación entre los tipos de contrato psicológico que se involucran en el desempeño de los miembros de la empresa, así como la relación establecida por los diferentes roles dentro de la organización.

Un cliente interno comprometido apoya y acepta los objetivos, la filosofía y los valores de la organización; está dispuesto a realizar un esfuerzo relevante en beneficio de la misma; y al estar motivado, el cliente interno deseará mantenerse dentro de la organización el mayor tiempo posible, para realizar su labor en busca de alcanzar los objetivos organizacionales planteados (Pérez Vera; De la Garza Carranza, 2008). Mientras que, para Bekin (2004), el marketing interno tiene como objetivo utilizar el conocimiento para que la empresa alcance sus objetivos competitivos en el escenario que la rodea, pero dicho conocimiento debe ser compartido entre los miembros de la misma; pues para satisfacer las necesidades del cliente externo se debe primero impulsar que el cliente interno se sienta satisfecho, valorado y feliz.

La función del marketing interno es mostrar que en los procesos dentro de una organización existe un cliente interno con valores específicos, por lo que esas ideas se quedan en la mente de los mismos, quienes serán beneficiados además del cliente externo. En este sentido, Silva y Lira (2014) señalan que con el uso de las acciones de marketing interno habrá mejoras en la calidad de los productos y servicios de la organización, con el diferencial del compromiso, de la productividad personal, que a su vez perfecciona los procesos dentro de la organización. 


\section{REFERENCIAS}

ALLEN, Natalie Jean; MEYER, John P. Affective, continuance, and normative commitment to the organization: an examination of construct validity. Journal of Vocational Behavior,Amsterdam, v.49, n.3, p.252-276, 1996.

ANTUNES PAES, Taís Alexander; ANTUNES PAES, Neir. A atividade turística na Baía da Traição e sua relação com o endomarketing. Turis Nostrum, João Pessoa, v.1, n.1, p.1-30, 2012.

ARCINIEGA, Luis. Compromiso organizacional en México ¿Cómo hacer que la gente se ponga la camiseta?Dirección Estratégica, México, DF, n.11, p.21-23, 2002.

BEKIN, Saul Faingaus. Endomarketing: como praticá-lo com sucesso. São Paulo: Prentice Hall, 2004.

BERRY, Leonard Len; HENSEL, James S.; BURKE, Marian C. Improving retailer capability for effective consumerism response. Journal of Retailing,New York, v.52, n.3, p.3-14, 1976.

BOHNENBERGER, Maria Cristina. Marketing interno: la actuación conjunta entre recursos humanos y marketing en busca del compromiso organizacional. 2005. Tese (Doutorado em Economia) - Universitat de les Illes Balears, Palma de Mallorca. 2005.

BRANDÃO, Nuno Goulart. A comunicação interna estratégica como reforço da valorização das pessoas e seus níveis de engagement nas organizações. Media \& Jornalismo, Lisboa, v.18, n.33, p.91-102, 2018. Disponível em: https://bit.ly/2ywkwA4. Acesso em: 1 ago. 2019.

CASTILLO-VERGARA, Mauricio; ALVAREZ-MARIN, Alejandro; MATSUDA OTEIZA, Ken; ALVARADO, Natalia; CODOCEO, Maria José. Impacto del marketing interno en el desarrollo de innovación: la co-creación en el sector turístico de La Serena-Chile.Estudios y Perspectivas en Turismo,Buenos Aires, v.25, n.2, p.203-222, 2016. Disponível em: https://bit.ly/2Z78Lvx. Acesso em: 6 out. 2017.

COCA CARASILA, Andrés Milton. El concepto de marketing: pasado y presente. Revista de Ciencias Sociales (Ve), Maracaibo, v.14, n.2, p.391-412, 2008. Disponível em: https://bit.ly/2Z3oWdo. Acesso em:22 jul. 2019.

GEYSKENS, Inge; STEENKAMP, Jan-Benedict E. M.; SCHEER, Lisa K.; KUMAR, Nirmalya. The effects of trust and interdependence on relationship commitment: a trans-Atlantic study. International Journal of Research in Marketing,Amsterdam, v.13, n.4, p.303-317, 1996.

GRÖNROOS, Christian. Marketing y gestión de servicios: la gestión de los momentos de la verdad y la competencia en los servicios. Madrid: Ediciones Díaz de Santos. 1994.

HELLRIEGEL, Don; SLOCUM JUNIOR. John W. Comportamiento organizacional. 10. ed. México, DF: Thompson, 2004.

HERNÁNDEZ SAMPIERI, Roberto; FERNÁNDEZ COLLADO, Carlos; BAPTISTA LUCIO, Pilar.Metodología de la investigación. 5. ed. México, DF: McGrawHill, 2010.

KOHLI, Ajay Kumar; JAWORSKI, Bernard J. Market orientation: the construct, research propositions, and managerial implications. The Journal of Marketing, Chicago, v.54, n.2, p.1-18, 1990. 
LOZANO RAMÍREZ, Ma. Cruz. El marketing interno como proceso de aprendizaje organizacional. Revista Internacional Administración \& Finanzas, Hilo, v.9, n.2, p.87-97, 2016.

LU, Vinh Nhat; CAPEZIO, Alessandra; RESTUBOG, Simon Lloyd D.; GARCIA, Patrick R. J. M.; WANG, Lu. In pursuit of service excellence: investigating the role of psychological contracts and organizational identification of frontline hotel employees. Tourism Management,Amsterdam, v.56, p.8-19, 2016.

MENDOZA MOHENO, Jessica; HERNÁNDEZ CALZADA, Martín Aubert; TABERNERO URBIETA, Carmen. Retos y oportunidades de la investigación en marketing interno. Revista de Ciencias Sociales (Ve),Maracaibo, v.17, n.1, p.110-125, 2011. Disponível em: https://bit.ly/2XYwlJv. Acesso em: 19 jul. 2019.

MEYER, John P.; HERSCOVITCH, Lynne. Commitment in the workplace: toward a general model. Human Resource Management Review,Amsterdam, v.11, n.3, p.299-326, 2001.

MOLINA CARMONA, Edith.; HERNÁNDEZ, Leticia Franco H. G. Manual para la elaboración de tesis. México, DF: Benemérita Universidad Autónoma de Puebla, 2006.

MOWDAY, Richard T.; STEERS, Richard M.; PORTER, Lyman W. The measurement of organizational commitment. Journal of Vocational Behavior,Amsterdam, v.14, n.2, p.224-247, 1979.

PARASURAMAN, A. Reflections on gaining competitive advantage through customer value. Journal of the Academy of Marketing Science,New York, v.25, n.2, 1997.

PÉREZ VERA, Rócio del Carmen; DE LA GARZA CARRANZA, María Teresa. Comportamiento organizacional y satisfacción laboral.Revista Raites,Guanajuato, v.2, n.3, p.100-108, 2008.

PETERSON, Douglas K.; PUIA, George M.; SUESS, Frederica R. Yo tengo la camiseta (I have the shirt on): an exploration of job satisfaction and commitment among workers in Mexico. Journal of Leadership and Organizational Studies, Newbury Park, v.10, n.2, p.73-88, 2003.

RAFIQ, Mohammed; AHMED, Pervaiz K. Advances in the internal marketing concept: definition, synthesis and extension. Journal of services marketing, Bingley, v.14, n.6, p.449-462, 2000.

RUIZALBA ROBLEDO, José Luis; VALLESPÍN ARÁN, María; PÉREZ-ARANDA, Javier. Gestión del conocimiento y orientación al marketing interno en el desarrollo de ventajas competitivas en el sector hotelero. Investigaciones Europeas de Dirección y Economía de la Empresa,Amsterdam, v.21, n.2, p.84-92, 2015.

SANTOS, Natália Contesini. A avaliação de resultados em comunicação interna: desafios e modelos de avaliação aplicáveis. Revista Foco, Vila Velha, v. 11, n. 1, p. 188-204, 2018.

SILVA, Sabrina; LIRA, Rodrigo Anido. Endomarketing como ferramenta de motivação: análise das suas aplicações em uma rede de supermercados no município de Campos dos Goytacazes-RJ. Perspectivas Online: Humanas Sociais \& Aplicadas, Campos dos Goytacazes, v.4, n.9, p.35-46, 2014. 
VILLAREJO RAMOS, Ángel F. La medición del valor de marca en el ámbito de la gestión de marketing.Sevilla: Ceade, 2002.

ZAPATA PALACIOS, Lelia; GÓMEZ QUIJANO, Arturo. El comunicador interno, de profesional de la información a profesional de la comunicación. In: Congreso Internacional Comunicación y Pensamiento. Comunicracia y Desarrollo Social, 1., 2016, Sevilla. Actas [...]. Sevilla: Universidad de Sevilla, 2016. p.540-562.

Artículo recibido el 08.02.2019 y aprobado el 12.07.2019. 